\title{
Measuring cortisol and DHEA in fingernails: A pilot study
}

This article was published in the following Dove Press journal:

Neuropsychiatric Disease and Treatment

16 December 2009

Number of times this article has been viewed

Fay Warnock'

Kevin McElwee ${ }^{2}$

Rubo J $\mathrm{Seo}^{3}$

Sean Mclsaac ${ }^{3}$

Danielle Seim ${ }^{3}$

Tatiana Ramirez-Aponte ${ }^{3}$

Karine AN Macritchie ${ }^{3}$

Allan $\mathrm{H}_{\text {Young }}^{3}$

'Developmental Neuroscience and Child Health, BC Children's Hospital, Vancouver, BC, Canada; ${ }^{2}$ Department of Dermatology and Skin Science, ${ }^{3}$ Institute of Mental Health-Department of Psychiatry, University of British Columbia, Vancouver, BC, Canada
Correspondence: Allan H Young Institute of Mental Health, Dept. of Psychiatry, University of British Columbia, Suite 403 - 5950 University Blvd., Vancouver, BCV6T IZ3, Canada

Tel + I 6048273685

$\mathrm{Fax}+\mathrm{I} 6048273373$

Email allan.young@ubc.ca
Purpose: Abnormalities in both cortisol and dehydroepiandrosterone (DHEA) have been reported in psychiatric disorders. Analysis of saliva, urine and blood cortisol and DHEA levels provides an index of hormone levels over a short time period. Unlike such conventional measures, fingernails incorporate endogenous hormones that passively diffuse to the nail matrix from capillaries during keratinization. This study piloted the measurement of cortisol and DHEA levels in fingernails as a potential measure of their accumulated secretion of steroid hormones over a prolonged time period.

Method: Thirty-three university students (18-24 years) provided fingernail samples on two occasions over a school semester. The visits were scheduled so nail cortisol and DHEA levels were collected from periods when students might be under different levels of stress.

Results: During the putatively stressful period, the nail samples showed a significant increase in the cortisol: DHEA ratio $(P=0.0002)$ due to a significant decrease in the DHEA levels $(P=0.004)$ and a numerical but not statistically significant increase in the cortisol levels $(P=0.256)$.

Discussion: This pilot study showed that nails can be used to measure cortisol and DHEA, a measure which may reflect environmental stress. More work is required to further validate this technique which may prove useful in studies of both healthy individuals and patient groups.

Keywords: stress, nails, cortisol, DHEA

\section{Introduction}

The human stress response is modulated in part by the hypothalamic-pituitary-adrenal (HPA) axis. Activation of the HPA axis induces the release of cortisol and related steroids such as dehydroepiandrosterone (DHEA) from the adrenal cortex. ${ }^{1}$ DHEA functions as a cortisol antagonist and may protect the brain from the negative effects of stress. ${ }^{2}$ The cortisol: DHEA ratio has been suggested as a measure of "functional hypercortisolemia". 3

Although well-established techniques measure levels of cortisol and DHEA using saliva, urine and blood, these only provide a measure of hormone levels over a short time period. Fingernail analysis, on the other hand, may allow assessment of the accumulated hormone levels over a longer time period as neutrally charged endogenous hormones passively diffuse from capillaries to the nail matrix and become incorporated into keratin during nail formation. ${ }^{4}$

In this study we measured cortisol and DHEA in nails obtained from university students at two different periods during the school year. Based on a notion that the cortisol: DHEA ratio may be used to assess "functional hypercortiolemia," we hypothesized that the cortisol: DHEA ratio would be higher in the nail keratinized during the periods of the school year perceived by the students to be more stressful. ${ }^{2,3}$ submit your manuscript | www.dovepress.com

Dovepress
Neuropsychiatric Disease and Treatment 2010:6 I-7

(C) 2010Warnock et al, publisher and licensee Dove Medical Press Ltd. This is an Open Access article which permits unrestricted noncommercial use, provided the original work is properly cited. 


\section{Material and methods}

\section{Participants}

The study was conducted in accordance with the Declaration of Helsinki. All procedures were approved by the local University Clinical Research Ethics Board and conducted with the informed and written consent of the subjects. To be included, students had to have no significant physical health problems, psychiatric disorders, endocrine disorders, neurological disorders, nail diseases, and to not be pregnant. Eligibility also required that students were on no medications likely to interfere with hypothalamic-pituitary-adrenal (HPA) axis function or the assay. Participants were first screened for initial eligibility criteria during email contact with a team member. Of 37 students who responded, three dropped out, and one was excluded because she was on corticosteroid medication. Thirty-three eligible undergraduate students (26 females, seven males: mean age of 20.8, standard deviation $[\mathrm{SD}]=1.6$, range $18-24$ years) were recruited. Two women were on combined oral hormone contraception.

\section{Sample collection and preparation}

All 33 study participants completed three visits. The first visit was in September 2008, the second visit (baseline) was in November 2008, and the third visit (experimental) was in March 2009. During the first visit in September, participants were further screened, provided demographic information and written consent to participate. Participants were instructed to clip their fingernails two weeks prior to the baseline and experimental visits so that samples collected at each of those visits would represent two weeks of accumulated stress. During the baseline and experimental visits, each participant was directed by the onsite monitor to provide fingernail samples from every digit by clipping directly into a Ziploc ${ }^{\circledR}$ bag to avoid losing any parts of the sample. The timing of the baseline and experimental visits was chosen according to the rate of nail growth; it takes an average of three months to grow from the nail matrix to become a free nail. ${ }^{4}$ The clipped nail samples obtained in the baseline visit represented the stress levels in August when students may be under less stress with relatively few academic obligations. The clipped nail samples obtained in the experimental visit represented the participants' stress levels in December when students are more likely to be stressed due to exams and term paper deadlines. These time points were also based on a previous survey on student stress reported by the University of Alberta in 2008. ${ }^{6}$ Nail samples from each participant were placed in labeled Ziploc ${ }^{\circledR}$ bags and stored at $-20^{\circ} \mathrm{C}$ until use.

\section{Nail hormone extraction}

Prior to testing of the main hypothesis, two methanol extraction methods were piloted in order to find the better extraction efficiency. Samples were randomly allocated to either method.

\section{Method I: Part nail extraction method}

Nail samples allocated to Method 1 were washed twice with $10 \mathrm{ml}$ of isopropanol and air-dried overnight. Isopropanol was collected. The dried nail sample was ground using a ball mill (Retsch ${ }^{\circledR}$, Haan, Düsseldorf, Germany) at $30 \mathrm{~Hz}$ for five minutes. Fifty milligrams of nail powder were transferred to a $1.7 \mathrm{ml}$ centrifuge tube. One milliliter of methanol was added, and the tube was rotated on a Labquake tube shaker (Fisher Scientific, Ottawa, Ontario, Canada) overnight at room temperature. After 24 hours, the sample was vortexed and centrifuged at 10,000 rpm for five minutes. Eight hundred microliters of supernatant were aliquoted to a clean tube and evaporated under a nitrogen gas stream until dry.

\section{Method 2: Whole nail extraction method}

In Method 2, allocated nail samples were vortex-washed twice with $5 \mathrm{ml}$ of isopropanol for one minute. Isopropanol was collected and the sample was dried overnight. The dried nail sample was ground using a ball mill at $25 \mathrm{~Hz}$ for 2.5 minutes. A clean $5 \mathrm{ml}$ polystyrene round-bottom tube (Falcon ${ }^{\circledR}$; Franklin Lakes, NJ, USA) was prepared and weighed. The whole ground nail powder was transferred to the tube and re-weighed to measure the difference. $1.5 \mathrm{ml}$ of methanol was added and the tube was rotated on a Labquake tube shaker overnight. The sample was centrifuged, and the whole volume of supernatant was aliquoted into a clean tube. Another $1.5 \mathrm{ml}$ of methanol was added to the sample tube and the whole extraction process was repeated again overnight. After completing the second extraction, $3 \mathrm{ml}$ of supernatant aliquoted from the first and second days of extractions was evaporated overnight under a fume hood.

For both methods, methanol from an additional extraction (not included in the protocol) and the collected isopropanol from the initial wash were analyzed in order to eliminate any chance of unaccounted cortisol or DHEA.

\section{EIA assay}

Evaporated samples were resuspended in assay diluent provided by the EIA manufacturer. Cortisol and DHEA were analyzed by EIA assay according to manufacturer's instructions (Salimetrics, Salivary Assay Cortisol/DHEA kit, Cat. No. 1-3002/1-1202, State College, PA, USA). The kit was 
chosen based on findings from previous studies on measuring cortisol levels in hairs; Salimetric's High Sensitive Salivary Cortisol/DHEA provides higher and wider range of detection window $(0.012 \mu \mathrm{g} / \mathrm{dL}<$ cortisol $<3.0 \mu \mathrm{g} / \mathrm{dL} ; 3.0 \mathrm{pg} / \mathrm{mL}$ $<$ DHEA $<1000 \mathrm{pg} / \mathrm{mL}$ ) than liquid chromatography with mass spectrometry, ELISA and CLIA. ${ }^{6-9}$ Ten duplicates were used per kit for the intra-assay coefficient of variation, and cortisol and DHEA controls were supplied by the assay manufacturer for the inter-assay coefficient of variation. The intra- and inter-assay variations for cortisol were $2.79 \%$ and $6.02 \%$, respectively. The inter-assay variation for DHEA was $7.90 \%$.

\section{Statistical analysis}

After assessment of the distribution of data using a Kolmogorov-Smirnov test, the Wilcoxon's signed rank test was applied to assess differences in the nail cortisol, DHEA and the cortisol: DHEA ratio between the baseline and experimental visits. The correlation between cortisol and DHEA nail concentrations from each visit was calculated using Spearman's rho.

\section{Results}

There was no significant difference in the levels of cortisol ( $\mathrm{U}=95.00, P=0.38)$ or DHEA ( $\mathrm{U}=131, P=0.88)$ using the two pilot extraction protocols. Cortisol was significantly correlated with DHEA at both visits $(P=0.001$ and 0.001 , $\mathrm{r}=0.510$ and 0.583 , respectively; Figures 1 and 2 ). The two women on combined oral hormone contraception were not outliers in any of these analyses. No traceable amounts of cortisol or DHEA were detected in methanol from the additional extraction or in isopropanol (Data not shown).

Cortisol and DHEA concentrations are summarized in Table 1 for the participants; two samples that were outliers were removed with the remaining 31 samples carried forward for further analyses. There was a significant decrease

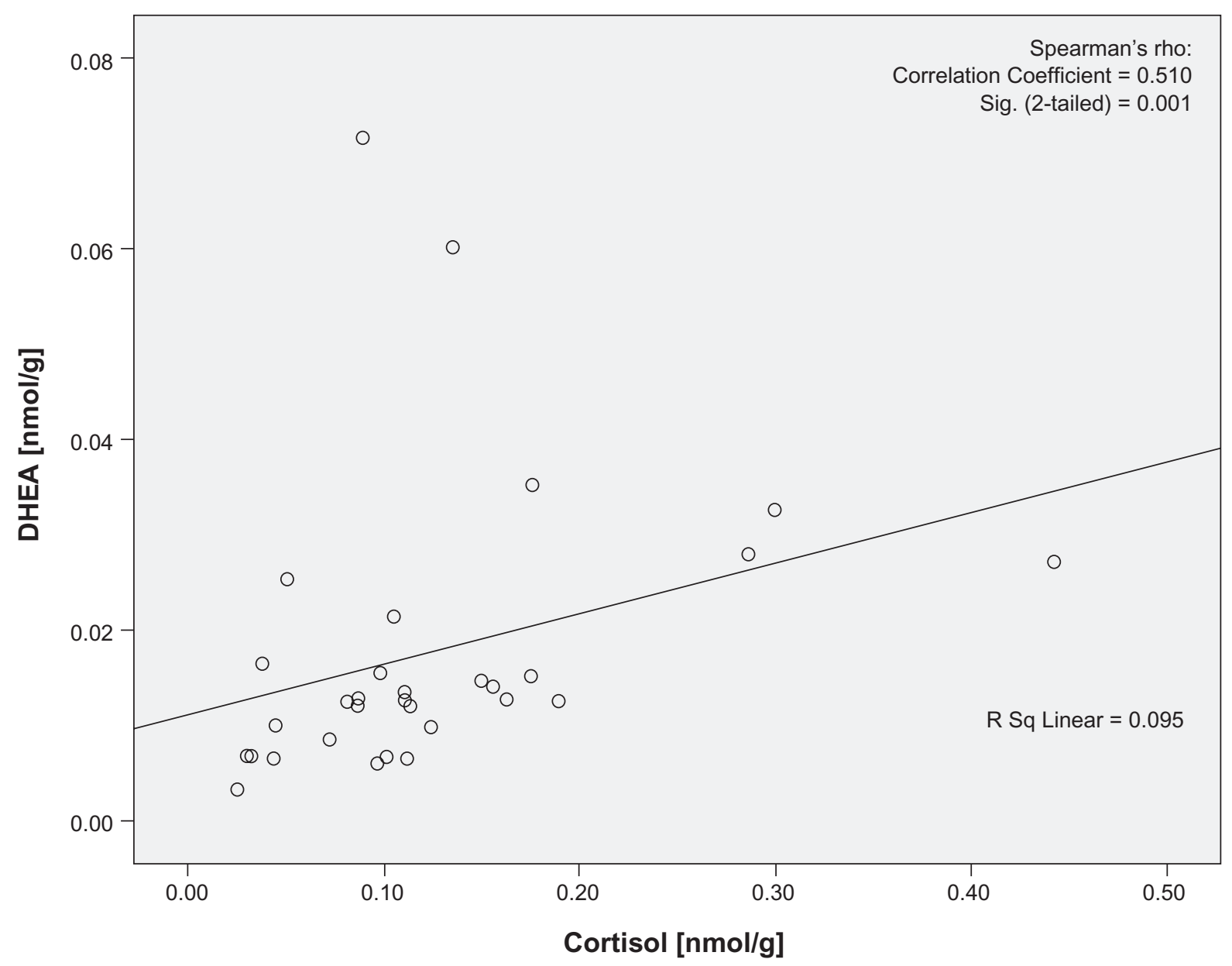

Figure I Correlation plots of the cortisol and DHEA concentrations at baseline visit. Cortisol denotes nail cortisol levels to the beginning of the school period and DHEA denotes the nail DHEA levels to the beginning of the school period. Note the statistically significant correlation between cortisol and DHEA in baseline visit. Abbreviation: DHEA, dehydroepiandrosterone. 


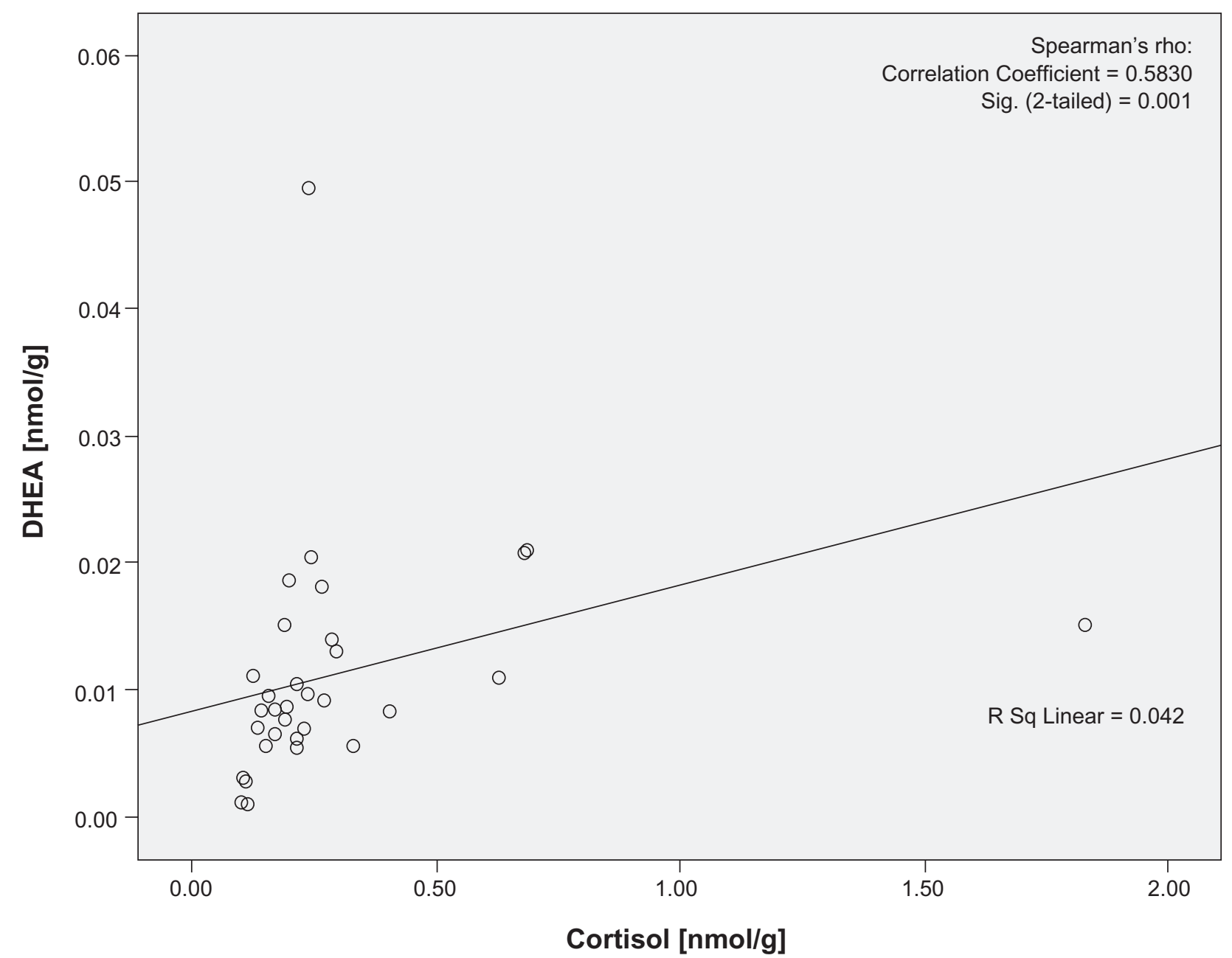

Figure 2 Correlation plots of the cortisol and DHEA concentrations at experimental visit. Cortisol denotes nail cortisol levels to the exam period and DHEA denotes the nail DHEA levels to the exam period. Note the statistically significant correlation between cortisol and DHEA in experimental visit. Abbreviation: DHEA, dehydroepiandrosterone.

Table I Distribution of age and hormone levels of participants who completed all three visits

\begin{tabular}{llll}
\hline & \multicolumn{3}{l}{ Descriptive statistic $(\mathbf{N}=\mathbf{3}$ I) } \\
\cline { 2 - 4 } & Mean (SD) & Median & Range \\
\hline Age & 20.8 & 21.0 & $18-24$ \\
$\begin{array}{l}\text { Baseline Cortisol } \\
\text { (nmol/g) }\end{array}$ & $0.1234(0.08857)$ & 0.1056 & $0.0253-0.4426$ \\
$\begin{array}{l}\text { Experiment Cortisol } \\
\text { (nmol/g) }\end{array}$ & $0.2119(0.3152)$ & 0.1406 & $0.0249-1.7523$ \\
$\begin{array}{l}\text { Baseline DHEA } \\
\text { (nmol/g) }\end{array}$ & $0.01773(0.01516)$ & 0.01271 & $0.03429-0.07163$ \\
$\begin{array}{l}\text { Experiment DHEA } \\
\text { (nmol/g) }\end{array}$ & $0.01174(0.08875)$ & 0.00943 & $0.01693-0.0504 I$ \\
$\begin{array}{l}\text { Cortisol: DHEA at } \\
\text { baseline visit }\end{array}$ & $8.5170(4.4765)$ & 8.1808 & $1.2453-17.1706$ \\
$\begin{array}{l}\text { Cortisol: DHEA at } \\
\text { experimental visit }\end{array}$ & $18.8093(19.6808)$ & $12.667 \mid$ & $3.3269-46.8723$ \\
\hline
\end{tabular}

Abbreviation: DHEA, dehydroepiandrosterone; SD, standard deviation. in the levels of DHEA $(P=0.004)$ at the experimental visit (mean $=0.01174 \mathrm{nmol} / \mathrm{g}, 95 \%$ confidence interval [CI]: $8.47 \mathrm{E}-6,1.48 \mathrm{E}-5)$ compared to the baseline visit $($ mean $=0.01773 \mathrm{nmol} / \mathrm{g}, 95 \%$ CI: $1.22 \mathrm{E}-5,2.29 \mathrm{E}-5)$ (Figure 3) as well as a significant increase in the cortisol: DHEA ratios $(P=0.0002)$ measured at the experimental visit (mean $=8.5170,95 \%$ CI: $12.00,25.98)$ compared to the baseline visit $($ mean $=18.8093,95 \%$ CI: $2.15,26.93$ ) (Figure 4). The change in mean cortisol level between the two visits increased marginally (mean $=0.1234 \mathrm{nmol} / \mathrm{g}$ and $0.2119 \mathrm{nmol} / \mathrm{g}, 95 \% \mathrm{CI}: 0.09,0.16$ and $0.10,0.32$, respectively) but was not statistically significant $(P=0.256)$ (Figure 5).

\section{Discussion}

This pilot study demonstrates the feasibility of measuring nail cortisol and DHEA levels. Other studies using saliva, urine, and blood samples have measured cortisol and DHEA 


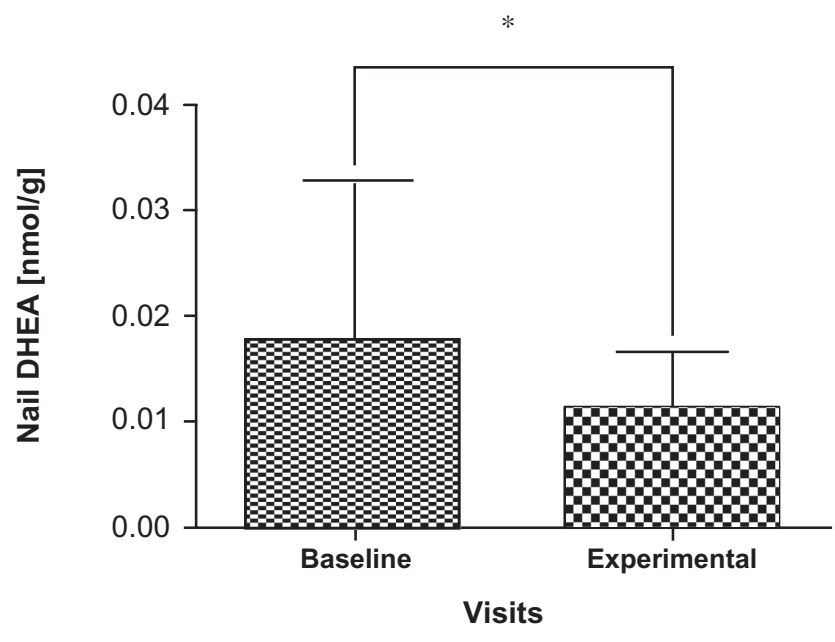

Figure 3 Comparison of mean and standard deviation in nail DHEA levels from baseline visits and experimental visits. Baseline denotes nail DHEA levels to the beginning of the school period and experimental denotes the nail DHEA levels to the exam period. Note the decrease in DHEA levels in experimental visits which show statistically significant changes from baseline visits.

Note: *Indicates $P<0.0$ I Wilcoxon's signed rank test.

Abbreviation: DHEA, dehydroepiandrosterone.

levels as a valid measure of short term HPA activity., 3,5,6,12,13 However, methods using saliva, urine, blood and hair have their own limitations. Saliva collection is potentially influenced by medications or food ingredients and is under the influence of diurnal changes. ${ }^{14}$ Urine collection is the most difficult method for subjects to comply with and its validity is dependent on a complete 24-hour collection. Collecting blood is invasive and potentially anxiogenic. ${ }^{15}$ Samples collected

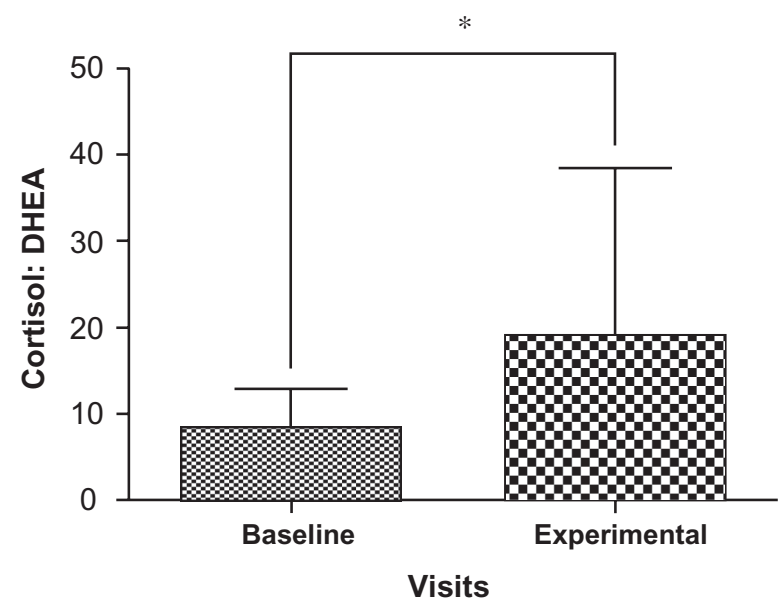

Figure 4 Comparison of mean and standard deviation in cortisol:DHEA ratios from baseline visits and experimental visits. Baseline denotes nail cortisol: DHEA ratios to the beginning of the school period and experimental denotes nail cortisol:DHEA ratios to the exam period. Note the increase in cortisol: DHEA ratios in experimental visits which show statistically significant changes from baseline visits.

Note: *Indicates $P<0.0$ I Wilcoxon's signed rank test.

Abbreviation: DHEA, dehydroepiandrosterone. from these established methods also need to be kept frozen at $-20{ }^{\circ} \mathrm{C}$ before the assay to prevent interaction between an enzyme, 17- $\beta$-hydroxysteroid dehydrogenase (17 $\beta$-HSD), and its substrate, DHEA; $17 \beta$-HSD is a ubiquitous enzyme involved in conversion of DHEA into its derivative, androstenediol. Once the conversion occurs, the EIA assay can no longer detect the original DHEA levels. On the other hand, endogenous steroid hormone extraction and detection from hairs and fingernails may eliminate this issue of unaccounted hormonal derivatization due to their solid media, keratin by physically blocking the access of $17 \beta$-HSD to DHEA even at room temperature as opposed to the established methods with liquid media. Hair analysis has previously been employed for the identification of long term hormonal levels. However, hair collection is unacceptable to some patients for religious or socio-cultural reasons, and samples may be contaminated by sweat. ${ }^{15,16}$ In contrast, nail clipping is more acceptable in most cultures and nails are known for their resistance to decomposition and disintegration. ${ }^{16}$ Further studies are also recommended pertaining to the stable preservation of steroid hormones in nails.

In order to find better extraction efficiency, two nail steroid extraction methods were piloted. There was no significant difference between these two methods. However, nail powder is in the solid phase from which homogeneity can be hardly attained. Thus, taking a random $50 \mathrm{mg}$ of nail powder as per the part nail extraction method is less likely to be an accurate reflection of the exact concentrations of the accumulated steroid hormones produced during the period of interest. The use of the whole nail powder as described in the whole nail extraction method is recommended in order to better contain the accumulated endogenous steroid hormones secreted during the period of interest.

This study is in part based on the notion that the nail samples were representative of the period three months prior to clippings. ${ }^{4}$ However, the nail growth rate varies with seasonal changes, sex, different finger digits, age, clipping frequency, nail filing, and nail-biting habits. ${ }^{16}$ Controls to such variables need to be employed for sampling in order to establish the relevance of variables to this technique.

As hypothesized, we found significant elevation in the cortisol: DHEA ratio from the nail samples collected from the experimental visit. This corresponded to a survey on stress levels conducted on students by the University of Alberta in 2008. ${ }^{6}$ However, the difference in cortisol: DHEA ratio is not because of a statistically significant increase in cortisol levels but rather because of significant decrease in DHEA levels. 


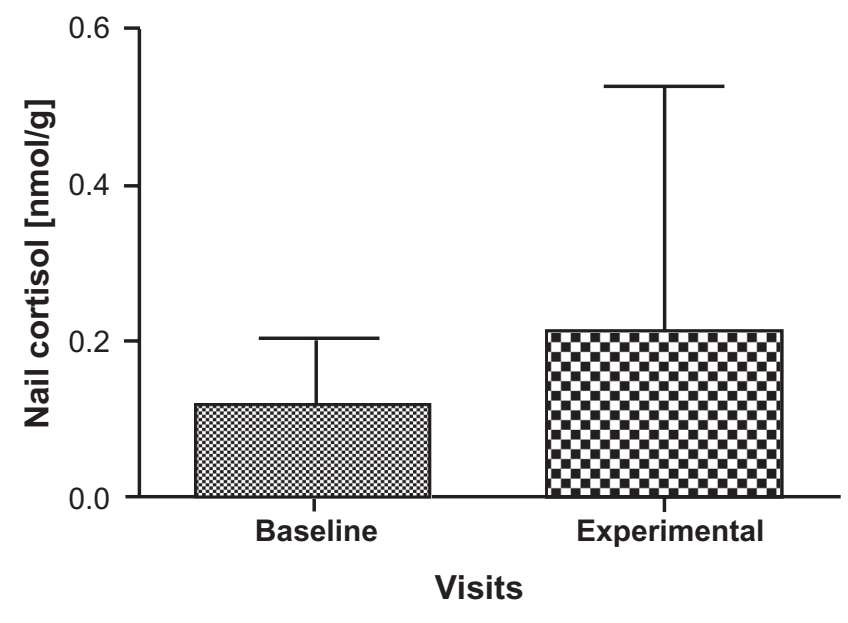

Figure $\mathbf{5}$ Comparison of mean and standard deviation in Nail cortisol levels from baseline visits and experimental visits. Baseline denotes nail cortisol levels to the beginning of the school period and experimental denotes the nail cortisol levels to the exam period. A numerical increase in cortisol levels was noted in experimental visits but not statistically significant from baseline visits.

Unfortunately, we did not employ an independent measure of analyzing matrix or interference effect for the resuspension medium but used the one which was provided by the EIA kit manufacturer. As mentioned earlier, the kit was chosen in light of findings from previous studies on measuring cortisol levels in hairs which also used methanol as an extractant and assay diluent as a resuspension medium. There may have been other better options of extractants and resuspension media to choose from. However, the current study is not looking at the absolute cortisol level but the ratios between cortisol and DHEA where both hormones were extracted and resuspended in identical manners. In other words, our study is not aiming to get the absolute cortisol levels but rather more normalized hormonal ratios. Considering that the identical extraction and resuspension efficiency were applied to both hormones, cortisol: DHEA ratio provides more normalized indices that pose more significance and relevance to measure the HPA axis activity.

In this study, we did not utilize other measurements of cortisol and DHEA secretion to compare differing indices of HPA axis activity over the period concerned. It has been reported that hair cortisol, another chronic measure of steroid hormones, is highly correlated to salivary cortisol in response to stress caused by relocation among a rhesus macaque population. ${ }^{17}$ However, it has also been reported in human subjects that hair cortisol is not statistically correlated to salivary cortisol but does correlate with urine cortisol. ${ }^{18}$ Although the human study is more directly relevant, it is clear that definitive data concerning these different indices of HPA axis activity are not yet available. Cross-validation of cortisol and DHEA levels between hair and nail samples should also be done.
This comparison, however, is complicated for both methods must take into account two different growth rates.

Another weakness of this study was the unequal sex distribution (24 females and seven males). We also did not monitor the women's menstrual phase. This might explain our failing to find significantly elevated cortisol levels during the stressful period as cortisol levels are more variable in females during the luteal phase than during the follicular phase. ${ }^{19}$ Notably, we did not specifically control for body mass index (BMI). According to Fraser and colleagues, urinary cortisol levels show a positive correlation with BMI. ${ }^{20}$ Cross-validation with an established cortisol and DHEA analysis method may be appropriate in order to categorize our participants as more "functionally hypercortisolemic" during the experimental visit than the baseline visit.

In conclusion, the finding that nails may yield a viable measure of cortisol and DHEA levels has important implications. This is a practicable method of measuring cortisol: DHEA ratios over a prolonged time period in human populations. The method could be useful in circumstances when an assessment of cortisol: DHEA ratio beyond acute periods is needed such as in chronic illness, doping tests, pregnancy, or postmortem studies. Future studies should further investigate the validity of the technique by establishing normative values and assessing its applicability in clinical settings.

\section{Acknowledgments/disclosures}

Dr Allan H Young and Dr Fay Warnock designed the study, developed the protocol, and edited the paper. Dr Kevin McElwee provided vital information in developing the protocol and commented on the paper. Mr Rubo Jiwon Seo developed the lab protocol, carried out the lab works, wrote the first draft of the manuscripts. Mr Sean McIsaac designed the study and recruited participants. Ms Danielle Seim managed participants' visits and collected samples. Ms Tatiana Ramirez-Aponte managed the literature searches and wrote the first draft of the manuscripts. Dr Karine AN Macritchie and Rubo Jiwon Seo undertook the statistical analysis and edited the paper. All authors contributed to and have approved the final manuscript. All authors declare that they have no conflicts of interest in this work. Funding for this study was provided by UBC HeRRO received from the Michael Smith Foundation for Health Research (MSFHR); the MSFHR had no further role in study design; in the collection, analysis and interpretation of data; in the writing of the report; and in the decision to submit the paper for publication. We thank Naomi Tagawa and Mukai Yuiko from Showa University School of Medicine, Japan, for their outstanding 
assistance with the Cortisol and DHEA extraction and the further EIA assays.

\section{References}

1. Young AH. Cortisol in mood disorders. Stress. 2004;7(4):205-208.

2. Jogems-Kosterman BJ, de Knijff DW, Kusters R, van Hoof JJ. Basal cortisol and DHEA levels in women with borderline personality disorder. J Psychiatr Res. 2007;41(12):1019-1026.

3. Young AH, Gallagher P, Porter RJ. Elevation of the cortisoldehydroepiandrosterone ratio in drug-free depressed patients. $\mathrm{Am}$ J Psychiatry. 2002;159(7):1237-1239.

4. de Berker DA, Andre J, Baran R. Nail biology and nail science. Int J Cosmet Sci. 2007;29(4):241-275.

5. Shirotsuki K, Izawa S, Sugaya N, et al. Salivary cortisol and DHEA reactivity to psychosocial stress in socially anxious males. Int $J$ Psychophysiol. 2009;72(2):198-203.

6. Markopoulou K, Papadopoulos A, Juruena MF, Poon L, Pariante CM, Cleare AJ. The ratio of cortisol/DHEA in treatment resistant depression. Psychoneuroendocrinology. 2009;34(1):19-26.

7. University of Alberta. Alberta: Student Counselling Services; c2002-09 [updated 2002]. Available from: http://www.uofaweb.ualberta.ca/ counselling/execsummary.cfm. Accessed May 3, 2009.

8. Raul JS, Cirimele V, Ludes B, Kintz P. Detection of physiological concentrations of cortisol and cortisone in human hair. Clin Biochem. 2004;37:1105-1111.

9. Sauvé B, Koren G, Walsh G, et al. Measurement of cortisol in human hair as a biomarker of systemic exposure. Clin Invest Med. 2007;30(5): E183-E191.

10. Kirschbaum C, Tietze A, Skoluda N, Dettenborn L. Hair as a retrospective calendar of cortisol production-Increased cortisol incorporation into hair in the third trimester of pregnancy. Psychoneuroendocrinology. 2009;34(1):32-37.
11. Davenport MD, Tiefenbacher S, Lutz CK, Novak MA, Meyer JS. Analysis of endogenous cortisol concentrations in the hair of rhesus macaques. General and Comparative Endocrinology. 2006;147: 255-261.

12. Goodyer IM, Herbert J, Tamplin A. Psychoendocrine antecedents of persistent first-episode major depression in adolescents: A communitybased longitudinal enquiry. Psychol Med. 2003;33(4):601-610.

13. Yehuda R, Brand SR, Golier JA, Yang RK. Clinical correlates of DHEA associated with post-traumatic stress disorder. Acta Psychiatr Scand. 2006;114(3):187-193.

14. Van Uum SH, Sauve B, Fraser LA, Morley-Forster P, Paul TL, Koren G. Elevated content of cortisol in hair of patients with severe chronic pain: A novel biomarker for stress. Stress. 2008;11(6):483-488.

15. Ice GH, James GD, eds. Measuring stress in humans: A practical guide for the field. New York: Cambridge University Press; 2007.

16. Gupta GR, Dhruw VK, Athawal BK, et al. Human nail growth pattern and medicolegal aspect. JIAFM. 2005;27(2):971-973.

17. Davenport MD, Tiefenbacher S, Lutz CK, Novak MA, Meyer JS. Analysis of endogenous cortisol concentrations in the hair of rhesus macaques. Gen Comp Endocrionol. 2006;147:255-261.

18. Sauvé B, Koren G, Walsh G, Tokmakejian S, Van Uum SH. Measurement of cortisol in human hair as a biomarker of systemic exposure. Clin Invest Med. 2007;30(5):E183-E191.

19. Symonds CS, Gallagher P, Thompson JM, Young AH. Effects of the menstrual cycle on mood, neurocognitive and neuroendocrine function in healthy premenopausal women. Psychol Med. 2004;34(1) 93-102.

20. Fraser R, Ingram MC, Anderson NH, Morrison C, Davies E, Connell JM. Cortisol effects on body mass, blood pressure, and cholesterol in the general population. Hypertension. 1999;33(6):1364-1368.
Neuropsychiatric Disease and Treatment

\section{Publish your work in this journal}

Neuropsychiatric Disease and Treatment is an international, peerreviewed journal of clinical therapeutics and pharmacology focusing on concise rapid reporting of clinical or pre-clinical studies on a range of neuropsychiatric and neurological disorders. This journal is indexed on PubMed Central, the 'PsycINFO' database and CAS, and is the official

\section{Dovepress}

journal of The International Neuropsychiatric Association (INA). The manuscript management system is completely online and includes a very quick and fair peer-review system, which is all easy to use. Visit http://www.dovepress.com/testimonials.php to read real quotes from published authors. 